\title{
Avaliação de manifestações dolorosas em pacientes internados em hospital de referência, com diagnóstico provisório de dengue*
}

\author{
Evaluation of pain manifestations in patients admitted to a reference hospital with \\ presumptive dengue fever diagnosis
}

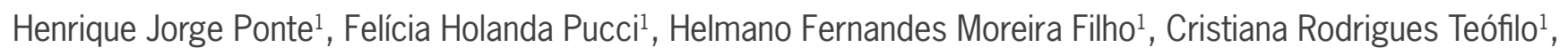
Roberto da Justa Pires Neto ${ }^{2}$

${ }^{*}$ Recebido do Hospital São José de Doenças Infecciosas (HSJ). Fortaleza, CE.

\section{RESUMO}

JUSTIFICATIVA E OBJETIVOS: As manifestações álgicas são características marcantes da dengue, tanto em crianças como em adultos, trazendo desconforto e sofrimento aos pacientes. O objetivo deste estudo foi analisar as principais queixas dolorosas em pacientes acometidos por essa afecção, tornando possível a elaboração de medidas de diagnóstico precoce e manuseio da dor.

MÉTODO: Estudo retrospectivo em 252 prontuários de pacientes com diagnóstico presuntivo de dengue, atendidos no Hospital São José de Fortaleza, referência em doenças infecciosas no estado do Ceará, no período de janeiro de 2006 a dezembro de 2007. Foram incluídos no estudo os pacientes que apresentaram ao menos uma queixa álgica.

RESULTADOS: A maioria dos pacientes (94,84\%) apresentou ao menos uma queixa álgica, sendo a cefaleia a mais prevalente, seguida por mialgia e dor abdominal. Os pacientes diagnosticados com febre hemorrágica da dengue $(40,16 \%)$ cursaram com mais sintomas dolorosos que os pacientes com diagnóstico de dengue clássico. Sorologia para dengue foi positiva em $72,72 \%$ dos que apre-

1. Graduando do Curso de Medicina da Universidade de Fortaleza (UNIFOR). Fortaleza, CE, Brasil.

2. Professor do Curso de Medicina da Universidade Federal do Ceará (UFC); Médico Infectologista. Fortaleza, CE, Brasil.

Endereço para correspondência:

Henrique Jorge Ponte

Rua Coronel Jucá, 315/404 - Meireles

60170-320 Fortaleza, CE.

Fones: (085) 8808-6272 - (085) 9975-5917

E-mail: henriqueponte@uol.com.br sentaram as cinco principais queixas álgicas analisadas no estudo.

CONCLUSÃO: A dor mostrou-se bastante prevalente no quadro clínico da dengue, cabendo aos profissionais de saúde o manuseio adequado da sintomatologia álgica.

Descritores: Cefaleia, Dengue, Dor, Febre hemorrágica da dengue.

\section{SUMMARY}

BACKGROUND AND OBJECTIVES: Pain manifestations are major dengue fever characteristics, both in adults and children, bringing discomfort and distress to patients. This study aimed at analyzing major pain complaints in dengue fever patients, thus making possible the development of early diagnosis and pain management.

METHOD: Retrospective study of 252 medical records of patients with presumptive dengue fever diagnosis, treated at São José Hospital, Fortaleza, reference in infectious diseases in the State of Ceará, from January 2006 to December 2007. Patients presenting at least one pain complaint were included in the study.

RESULTS: Most patients (94.84\%) had at least one pain complaint, being headache the most prevalent, followed by myalgia and abdominal pain. Patients diagnosed with hemorrhagic dengue fever $(40.16 \%)$ had more pain complaints than patients with classic dengue fever. Serology for dengue fever was positive for $72.72 \%$ of patients presenting the five major pain complaints evaluated in this study. CONCLUSION: Pain was highly prevalent in dengue fever cases and health professionals should adequately manage pain symptoms.

Keywords: Dengue fever, Headache, Hemorrhagic dengue fever, Pain. 


\section{INTRODUÇÃO}

A dengue é uma das mais importantes doenças tropicais da atualidade. Sua prevalência vem aumentando drasticamente no mundo nos últimos anos, sendo considerado grave problema de saúde pública internacional. A Organização Mundial de Saúde (OMS) estima que $2 / 5$ da população mundial estejam em risco de contrair a doença e que ocorram mais de 50 milhões de novas infecções anualmente ${ }^{1}$.

Ocorre, sobretudo, em áreas tropicais e subtropicais e consiste em doença febril aguda, transmitida por mosquitos hematófagos, pertencente ao gênero Flavivirus e à família Flaviridae, e causada por quatro subtipos antigenicamente distintos do vírus da dengue $^{2}$ (DENV): DENV-1, DENV-2, DENV-3 e DENV4. O mosquito Aedes aegypti é o principal vetor e o verdadeiro reservatório ${ }^{3}$. Recentemente, o DENV-4 foi detectado em território brasileiro 25 anos após sua última notificação ${ }^{4}$, fazendo do Brasil um país com circulação simultânea dos quatro sorotipos da doença.

A infecção por dengue causa uma doença cujo espectro inclui desde formas clinicamente inaparentes, que seriam identificadas apenas com exames laboratoriais, até quadros graves de hemorragia e choque podendo evoluir para óbito ${ }^{5}$. Estes sintomas podem variar de acordo com a idade do paciente, em que os recém-nascidos e crianças geralmente desenvolvem febre com eritema, enquanto adolescentes e adultos frequentemente apresentam um quadro clínico de início abrupto com febre alta, dor retro-orbitária, mialgia, artralgia e cefaleia ${ }^{6}$. Em virtude desta alta morbimortalidade, a dengue tem sido alvo de inúmeras campanhas para o seu controle em todo o país.

Em áreas endêmicas, a dengue é uma das causas mais comuns de internação, especialmente, com manifestações dolorosas, sendo conhecida por "febre quebra-ossos", devido à prostração, mialgia e artralgia intensas ${ }^{7}$. Ela apresenta ainda outros sintomas de caráter álgico, como cefaleia, dor ocular e abdominal, dentre outras. Este perfil tem representado um desafio aos profissionais de saúde que atendem esses pacientes, principalmente, nas unidades de emergência, uma vez que devem proporcionar analgesia e suporte adequados.

O objetivo deste estudo foi analisar as principais queixas dolorosas em pacientes acometidos por essa afecção, tornando possível a elaboração de medidas de diagnóstico precoce e manuseio da dor.

\section{MÉTODO}

Após aprovação pelo Comitê de Ética e Pesquisa do Hospital São José de Doenças Infecciosas (HSJ), sob o protocolo $n^{\circ} 035 / 2004$, na cidade de Fortaleza, realizou-se este estudo retrospectivo, em pacientes com diagnóstico provisório de dengue, internados no período de janeiro de 2006 a dezembro de 2007, que apresentavam ao menos uma das seguintes queixas álgicas: cefaleia, mialgia, artralgia, dor ocular e dor abdominal. Os pacientes foram classificados pela classificação preconizada pelo Ministério da Saúde (MS).

Os dados foram submetidos à análise estatística coletados pelo programa Microsoft Access 2010 e os resultados foram dispostos no formato Microsoft Excel 2010.

\section{RESULTADOS}

Foram internados 252 pacientes com diagnóstico provisório de dengue, no período do estudo. Dos pacientes que apresentaram pelo menos uma queixa álgica $(94,8 \%)$, a maioria era do sexo feminino e a média geral de idade de $27,4 \pm 17,7$ anos, variando entre 10 meses a 90 anos.

A principal queixa álgica foi cefaleia $(79,9 \%)$, seguida por mialgia $(78,6 \%)$ e dor abdominal $(66,9 \%)$; dor ocular $(33,8 \%)$ e artralgia $(19,2 \%)$; entretanto, este perfil pode representar um viés de aferição, visto que em cerca de $70 \%$ dos prontuários destes pacientes não havia dados suficientes sobre a prevalência desses sintomas (Gráfico 1).

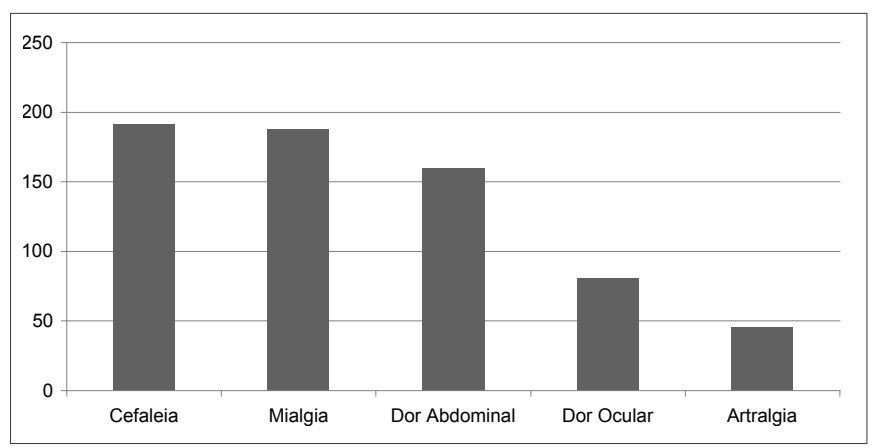

Gráfico 1 - Principais queixas álgicas em pacientes internados com diagnóstico provisório de dengue.

Apenas 11 pacientes evoluíram com todas as cinco queixas analisadas; 10 destes foram submetidos a testes para confirmação sorológica de dengue, sendo o exame positivo $(\mathrm{IgM}+)$ em oito pacientes. Outros 89 pacientes também apresentaram sorologia positiva, sendo maioria $\mathrm{o}$ grupo daqueles com prevalência de três sintomas álgicos 
simultâneos; o teste foi negativo em 31 pacientes e 107 não foram submetidos ao teste (Gráfico 2). Em relação ao desfecho, 221 pacientes receberam alta, 15 foram transferidos para outro hospital e 3 evoluíram para óbito.

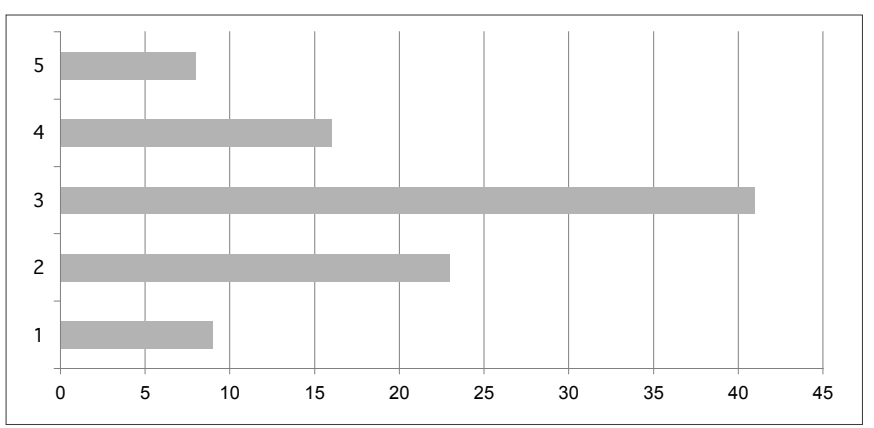

Gráfico 2 - Distribuição pelo número de sintomas dolorosos dos pacientes com sorologia positiva (IgM+).

Noventa e seis pacientes foram diagnosticados com febre hemorrágica da dengue, forma mais grave da doença, e, estes, cursaram com mais sintomas dolorosos que os 101 diagnosticados ao final com dengue clássica. Não houve análise de intensidade dolorosa com quaisquer escalas de avaliação da dor. Os demais 42 pacientes deixaram o hospital com outro diagnóstico ou o laudo final foi inconclusivo.

\section{DISCUSSÃO}

Depois de inoculada em humanos, através da picada da fêmea do vetor, a população viral inicial multiplica-se dentro de linfonodos regionais e entra na circulação sanguínea. $\mathrm{O}$ vírus tem um segundo ciclo de replicação, nesse momento dentro de monócitos, macrófagos e células musculares, explicando a intensa mialgia descrita em vários casos ${ }^{3}$.

A replicação viral induz a produção de citocinas por parte dos macrófagos, como o fator de necrose tumoral alfa (TNF- $\alpha$ ) e a interleucina (IL)6. A partir da liberação destas citocinas é que, provavelmente, dá-se o início do mal estar e quadro febril no paciente ${ }^{3,7}$. Do mesmo modo, tais substâncias estão fortemente relacionadas ao mecanismo de desenvolvimento de dor inflamatória, diminuindo o limiar álgico, causando hipernocicepção ${ }^{8}$.

A cefaleia, geralmente de localização frontal, e a dor retro-orbitária podem estar muitas vezes associadas a sintomas otorrinolaringológicos, simulando outras doenças, como quadro de sinusite aguda ${ }^{7}$. A cefaleia é uma manifestação geral frequente, atingindo $93 \%$ dos casos ${ }^{1,9}$. No presente estudo, foi sintoma registrado em $79,91 \%$ dos prontuá- rios. A cefaleia retro-orbitária pode estar relacionada com a multiplicação viral no tecido muscular adjacente, com comprometimento do nervo oculomotor ${ }^{9}$. Sintomas oculares são descritos em $30 \%$ dos casos $^{6}$, dado congruente com o encontrado no presente estudo $(33,89 \%)$.

Dos pacientes que evoluíram com todas as queixas álgicas analisadas, somente dois apresentaram sorologia negativa (IgM-) para dengue. Entretanto, um destes pacientes realizou o exame apenas dois dias após sua internação, conferindo, nesse caso, um possível viés, visto que os anticorpos IgM e IgG possuem diferentes períodos de picos e permanência. O primeiro pode ser observado a partir do $4^{\mathrm{o}}$ dia da doença, obtendo seu pico no fim da $1^{\mathrm{a}}$ semana. Já o último pode ser observado na $1^{\mathrm{a}}$ semana e tendo seu pico na $2^{\mathrm{a}}$ semana. Os anticorpos IgG podem continuar presentes por anos e são responsáveis pela imunidade de determinado sorotipo do vírus ${ }^{3}$. Logo, é inviável afirmar que este paciente, de fato, apresentava sorologia negativa para dengue, em razão da possibilidade do teste ainda não ter positivado.

Semelhante ao estudo que avaliou a epidemia de dengue ocorrida no Rio de Janeiro, nos anos de 2001 e 2002, as manifestações gerais da doença, como os sintomas dolorosos, tiveram alta ocorrência tanto na dengue clássica como na febre hemorrágica da dengue (FHD), não havendo diferença estatisticamente significativa, sendo esta de aproximadamente $4,4 \%$ maior na FHD no presente estudo. Isso pode ser justificado pela característica fisiopatológica do processo infeccioso, em que tais manifestações fazem parte do quadro clínico inicial, constituindo-se a maioria, inclusive, em critério diagnóstico para a doença ${ }^{10}$.

A doença pode se manifestar com outros sintomas, embora menos frequentes, como hepatomegalia dolorosa e dor epigástrica, dois indicadores de gravidade. Embora esta não seja critério para FHD, é comum, segundo a literatu$\mathrm{ra}$, associação com essa forma da doença $(38 \%-51 \%)^{11}$. Odinofagia pode estar presente e o diagnóstico diferencial com dengue torna-se importante ${ }^{7}$, pois o uso de medicamentos usuais, como anti-inflamatórios e salicilatos, pode agravar o processo hemorrágico ${ }^{1,5,7}$.

A dengue não tem tratamento específico. A medida terapêutica mais importante é a reposição hídrica, por via oral ou venosa nos casos mais graves. A febre e a artralgia devem ser tratadas com paracetamol ou dipirona em doses habituais. Em situações excepcionais, para pacientes com dor intensa, pode-se utilizar, nos adultos, a associação de paracetamol e fosfato de codeína ${ }^{5}$.

O paciente portador de dor intensa faz uso de vasto arsenal terapêutico, o que favorece o aparecimento de eventos 
adversos, como, por exemplo, a síndrome de Stevens-Johnson, no caso da associação descrita. Faz-se necessário que o paciente com suspeita ou diagnóstico de dengue seja, portanto, bem orientado quanto aos sintomas e à medicação que pode utilizar ${ }^{12}$.

\section{CONCLUSÃO}

Este estudo evidenciou a importância de uma abordagem ampla, principalmente com anamnese detalhada e exame físico minucioso a fim de identificar e manusear adequadamente as manifestações dolorosas em pacientes com dengue, devido a sua importância e prevalência no quadro clínico geral dessa doença.

\section{REFERÊNCIAS}

1. Guzmán MG, Kourí G. Dengue: an update. Lancet Infect Dis 2002;2(1):33-42.

2. Pessanha JE, Caiaffa WT, Cecilio AB, et al. Cocirculation of two dengue virus serotypes in individual and pooled samples of Aedes aegypti and Aedes albopictus larvae. Rev Soc Bras Med Trop 2011;44(1):103-5. 3. Lupi O, Carneiro CG, Coelho ICB. Manifestações mucocutâneas da dengue. An Bras Dermatol 2007;82(4):291-305.

4. Figueiredo RMP, Naveca FG, Bastos SM, et al. Dengue virus type 4, Manaus, Brazil. Emerg Infect Dis 2008;14(4):667-9.
5. Ministério da Saúde. Dengue - Diagnóstico e manejo clínico: adulto e criança. Secretaria de Vigilância em Saúde. 3a ed. Brasília/DF; 2007.

6. Dhasmana R. Bilateral premacular subhyaloid hemorrhage in dengue fever. J Ophthalmic Vis Res 2011;5(1):62-4.

7. Denis CK, Cavalcanti KM, Meirelles RC, et al. Manifestações otorrinolaringológicas em pacientes com dengue. Rev Bras Otorrinolaringol 2003;69(5):644-7. 8. Verri Jr WA, Cunha TM, Poole S, et al. Inibidores de citocinas e controle da dor. Rev Bras Reumatol, 2007;47(5):341-53.

9. Veronesi R. Tratado de infectologia. $3^{\mathrm{a}}$ ed. São Paulo: Atheneu; 2005. p. 343-55.

10. Casali CG, Pereira MRR, Santos LMJG, et al. A epidemia de dengue/dengue hemorrágico no município do Rio de Janeiro, 2001/2002. Rev Soc Bras Med Trop 2004;37(4):296-9.

11. Setiawan MW, Samsi TK, Wulur H, et al. Epigastric pain and sonographic assessment of the pancreas in dengue hemorrhagic fever. J Clin Ultrasound 1998;26(5):257-9.

12. Lobo JEO, Machado APM, Aguiar FC, et al. Síndrome de Stevens-Johnson em paciente com dor crônica. Relato de caso. Rev Dor 2009;10(4):370-3.

Apresentado em 12 de janeiro de 2011.

Aceito para publicação em 31 de maio de 2011. 